DEPÓSITO LEGAL ZU2020000153

Esta publicación científica en formato digital

es continuidad de la revista impresa

ISSN 0041-8811

E-ISSN 2665-0428

Revista

de la

Universidad

del Tunlia

Fundada en 1947

por el Dr. Jesús Emrique Lossada

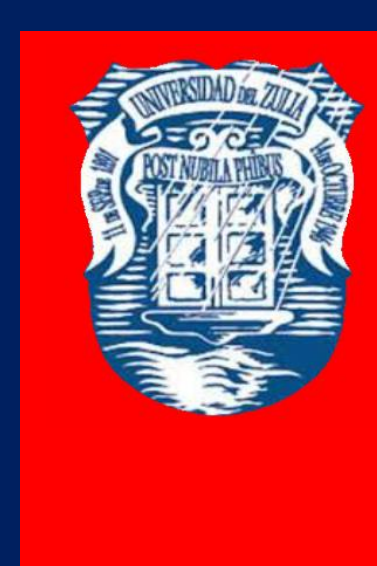

Ciencias

Sociales

y Arte

Aกัต 11 No 31

Septiembre - Diciembre 2021

Tercera ípoca

Maracailbo-Venezuela 
REVISTA DE LA UNIVERSIDAD DEL ZULIA. 3ª época. Año ll N 31, 2020

María del Pilar Quezada-Castro et al /// Desarrollo de competencias investigativas... 421-432

DOI: http://dx.doi.org/10.46925//rdluz.31.26

\title{
Desarrollo de competencias investigativas del estudiante de Derecho a partir del diseño de una rúbrica
}

\author{
María del Pilar Quezada-Castro* \\ María del Pilar Castro-Arellano ** \\ Juan Manuel Oliva-Núñez ${ }^{* * *}$ \\ Carlos Ignacio Gallo-Aguila **** \\ Guillermo Alexander Quezada-Castro*****
}

RESUMEN

El presente estudio analiza el uso de la rúbrica para desarrollar competencias investigativas en el estudiante de Derecho, la misma que es factible ser aplicada en las asignaturas de metodología de la investigación o tesis. El tema descrito es importante porque permite identificar las competencias investigativas que inciden en el proceso de formación de investigadores universitarios y proyectar la relevancia en el ejercicio profesional que otorgaría competitividad laboral. Se elaboró mediante el paradigma positivista, enfoque cuantitativo, diseño no experimental, tipo descriptivo y método analítico. A través del juicio de expertos y método Delphi se reconoce que la rúbrica holística promovería el pensamiento crítico, cultura científica, consolidación de semilleros de investigación con tendencia multidisciplinaria e interiorización del proceso de formación de investigadores desde las aulas universitarias, al ser una herramienta de autoevaluación.

PALABRAS CLAVE: Competencias investigativas, rúbrica holística, formación científica.

*Abogada. Docente en la Universidad Tecnológica del Perú. https://orcid.org/0000-0002-1012-570X. Email: C20853@utp.edu.pe

**Abogada. Coordinadora Académica de la Escuela Profesional de Turismo, Hotelería y Gastronomía de la Universidad Alas Peruanas, filial Piura - Perú. https://orcid.org/0000-0002-6661-9928. Email: m_castro_a@uap.edu.pe

** Doctor en Ciencias de la Educación. Coordinador de la oficina de Investigación, Innovación y Emprendimiento de la Universidad Alas Peruanas, Piura. Docente a tiempo completo en la Universidad Alas Peruanas, Perú - filial Piura. https://orcid.org/0000-0001-9319-3689. Email: j_oliva@doc.uap.edu.pe

**** Doctor en Ciencias de la Educación. Docente en la Universidad César Vallejo. https://orcid.org/0000-0003-1382-0545. Email: cgalloa@gmail.com

*****Abogado. Docente a tiempo completo en la Universidad Alas Peruanas, Perú -filial Piura. https://orcid.org/0000-0002-4868-1664. E-mail: guillermoquezadacastro@hotmail.com

Recibido: 14/07/2020

Aceptado: 02/09/2020 


\section{Development of investigative competences of the student of right from the design of a heading}

ABSTRACT

The present study analyzes the use of the rubric to develop investigative competences in the law student, the same that is feasible to be applied in the subjects of research methodology or thesis. The topic described is important because it allows to identify the investigative competences that affect the training process of university researchers and to project the relevance in the professional exercise that would grant labor competitiveness. It was elaborated using the positivist paradigm, quantitative approach, non-experimental design, descriptive type and analytical method. Through the expert judgment and Delphi method, it is recognized that the holistic rubric would promote critical thinking, scientific culture, consolidation of research hotbeds with a multidisciplinary tendency and internalization of the process of training researchers from university classrooms, as it is a self-assessment tool.

KEYWORDS: Investigative skills, holistic rubric, scientific training

\section{Introducción}

El proceso enseñanza-aprendizaje refleja, entre otros temas, el deber de formar estudiantes, a partir de conocimientos actualizados y útiles para su desenvolvimiento en la sociedad, es decir, no sólo representa la transmisión de contenidos ni la obtención de una calificación aprobatoria, puesto que ello no corresponde a la esencia de la disciplina denominada educación.

En la realidad peruana, a nivel de las facultades de Derecho, se percibe un aparente distanciamiento entre la producción científica y las asignaturas vinculadas a metodología de la investigación o tesis, situación que es de conocimiento público y se comprueba al revisar los repositorios institucionales de acceso libre, representando ello una inquietud para el equipo investigador.

Las asignaturas relacionadas a metodología de la investigación y tesis se caracterizan por la formación y motivación de los estudiantes; sin embargo, un factor innegable es la pretensión de reunir grupos numerosos alrededor de un docente y proyectarse que en un ciclo 
académico se logrará inculcar la pasión por investigar en las aulas, cuando ello tiene que ser enfocado de forma permanente.

De persistir en esta actuación, se advierten dos consecuencias reales: 1) la ausencia de producción científica a nivel de pregrado en las facultades de Derecho de las universidades peruanas y 2) la ausencia de habilidades investigativas en la formación de estudiantes de Derecho; ambas podrían originar una desventaja en la competitividad laboral.

En ese contexto, surge la interrogante ila rúbrica contribuiría al desarrollo de competencias investigativas del estudiante de Derecho?

Al respecto, Navarro et al (2019), sostienen que la rúbrica permite la corrección del trabajo elaborado por el estudiante y la evaluación de sus competencias, a través de la generación de dos tipos de rúbricas: las globales (aquellas que valoran con una visión general) y las analíticas (aquellas que valoran con una visión específica); ambas, según Alsina \& Rorua (2017) se presentan con una escala de progresividad y están determinadas hacia el desarrollo de una habilidad, conforme lo argumentan Araque-Marín et al (2019).

Por lo tanto, refieren Pacheco et al (2019), que su utilidad sí es necesaria en la educación superior, porque según Alcón et al (2017), sostienen que se caracteriza por un análisis que incluye medidas de acción destinadas a mejorar, ante lo cual agregan Rodríguez-Miñambres et al (2018), que facilita la comunicación entre docentes y estudiantes, toda vez que su contenido es formativo y puede ser considerado como una herramienta de autoevaluación, conforme lo sostienen Masero et al (2018).

Por su parte, Alcón (2016) manifesta que las rúbricas establecen el análisis de un concepto determinado, observando diferentes dimensiones, por lo que según Urias et al (2018), podría ser utilizado por el docente porque fomenta la participación activa del estudiante en su proceso de formación y evaluación, siendo necesario construir una rúbrica que permita la comprobación de los niveles de desempeño, conforme lo expresan Menéndez \& Gregori (2016).

Por lo tanto, contextualmente, la rúbrica ostentaría una aparente aplicación al caso específico; sin embargo, es neceario analizar su utilidad para establecer, determinar o reforzar competencias de los estudiantes de Derecho en su proceso de formación como investigadores. 
Ante lo cual, Castañeda et al (2018), sostienen que el término competencia está relacionado a un sistema, que contiene las destrezas necearias para una evaluación constante; siendo que en el caso de investigación, según García et al (2018), podrían determinarse a partir del reconocimiento de habilidades, por ejemplo: observación, descripción, análisis, síntesis e interpretación, representando ello, desde la perspectiva de Espinoza et al (2016), un reto para los educadores universitarios, toda vez que el desarrollo de competencias del estudiante incidirá en su formación profesional, lo cual permitirá la noble misión de presentar alternativas de solución ante los problemas de la realidad, conforme argumentan Martínez et al (2018).

En ese orden de ideas, García-Gutiérrez \& Aznar-Díaz (2019), agregan que la formación en investigación es un proceso vinculado a la enseñanza-aprendizaje, lo cual permitrá generar destrezas en el estudiante, desde un plano práctico y transversal, conforme lo consideran Veytia et al (2019), aunque para maximizar ello, refieren Parra et al (2019), es necesario la sistematización de experiencias tendientes al mejoramiento de la formación disciplinar en el estudiante.

El estudiante debe conceptualizar que la investigación está encaminada hacia la búsqueda de nuevos conocimientos, los cuales deberán ser comprobados, conforme lo señalan Segredo et al (2017), a través de la replicabilidad respectiva; ello permitirá, según Lorenzo et al (2017), que el estudiante interiorice la investigación científica en su formación como futuro egresado de la universidad, conforme lo sostienen Michalón et al (2019), aunado a lo que expresan Blanco et al (2019), beneficiará su personalidad propia.

De tal forma, que una vez que el estudiante interiorice la importancia de la investigación, Quezada et al (2020), consideran que es necessario desarrollar el pensamiento crítico como característica de la formación en competencias investigativas; claro que está que para lograr ello, Gonzáles \& Otero (2019) afirman que se debe priorizar la atención de tutoría a cargo de un docente, lo cual permitirá sentar bases para el autoaprendizaje constante, tal como lo consideran Vera-Rivero et al (2019) y complementando la idea, Cruz-Pérez et al (2019), expresan que es pertinente el uso y aprovechamiento de las tecnologías de información y 
REVISTA DE LA UNIVERSIDAD DEL ZULIA. 3a época. Año 11 N 31, 2020

María del Pilar Quezada-Castro et al /// Desarrollo de competencias investigativas... 421-432

DOI: http://dx.doi.org/10.46925//rdluz.31.26

comunicación, sin descuidar, lo que afirman Rodríguez et al (2019) que la actividad investigativa está relacionada con el respeto y observancia de los valores éticos empresariales.

\section{Metodología}

La presente investigación se caracteriza por ser paradigma positivista, enfoque cuantitativo, diseño no experimental, tipo descriptivo y método analítico.

El procedimiento utilizado para el diseño de la rúbrica consiste en:

1. Selección de expertos, quienes determinarán el tipo de rúbrica acorde a la naturaleza de la investigación.

2. Determinación de criterios de evalución y niveles de desempeño.

3. Identificación de las competencias.

4. Proyección de los resultados al aplicar la rúbrica en el estudiante de Derecho.

Respecto, a la selección de expertos, el equipo investigador convocó a 25 profesionales; sin embargo, debido al aislamiento social obligatorio derivado de la pandemia COVID-19, sólo fue posible conservar contacto fluído con 15, quienes se caracterizaron por ser Doctores en Educación, experiencia universitaria entre 7 a 13 años, vínculo laboral vigente con universidades peruanas y acercamiento directo con la asignatura de metodología de la investigación científica o tesis.

Los 15 expertos seleccionados brindaron validación de contenido a la rúbrica materia de la presente investigación y a través del método Delphi utiizado de manera online consideraron por mayoría que la rúbrica holística es ideal para el inicio de la formación de investigadores universitarios, porque se convierte en una herramienta de autoevaluación que le permita al estudiante de Derecho medir su avance en el proceso de elaboración de tesis, conforme se detalla en la tabla l que se muestra a continuación: 
REVISTA DE LA UNIVERSIDAD DEL ZULIA. 3ª época. Año ll N 31, 2020 María del Pilar Quezada-Castro et al /// Desarrollo de competencias investigativas... 421-432

DOI: http://dx.doi.org/10.46925//rdluz.31.26

Tabla 1. Rúbrica aplicable

\begin{tabular}{|c|c|c|c|c|}
\hline \multirow{2}{*}{$\begin{array}{l}\text { Criterios de } \\
\text { evaluación }\end{array}$} & \multicolumn{4}{|c|}{ Niveles de desempeño } \\
\hline & $\begin{array}{l}\text { Por mejorar } \\
\text { (0-5 puntos) }\end{array}$ & $\begin{array}{c}\text { Regular } \\
\text { (6-10 puntos) }\end{array}$ & $\begin{array}{c}\text { Bueno } \\
\text { (11-15 puntos) }\end{array}$ & $\begin{array}{c}\text { Excelente } \\
\text { (16-20 untos) }\end{array}$ \\
\hline $\begin{array}{l}\text { Idea de } \\
\text { investigación. }\end{array}$ & $\begin{array}{l}\text { El estudiante } \\
\text { no selecciona } \\
\text { una idea de } \\
\text { investigación. }\end{array}$ & $\begin{array}{lr}\text { El estudiante } \\
\text { selecciona } & \text { una } \\
\text { idea } & \text { de } \\
\text { investigación } & \text { sin } \\
\text { brindar } & \\
\text { explicación } & \text { oral } \\
\text { congruente. } & \end{array}$ & $\begin{array}{l}\text { El estudiante } \\
\text { selecciona una } \\
\text { idea de } \\
\text { investigación y } \\
\text { brinda explicación } \\
\text { oral congruente. }\end{array}$ & $\begin{array}{l}\text { El estudiante } \\
\text { selecciona una idea } \\
\text { de investigación, } \\
\text { brinda una } \\
\text { explicación oral y } \\
\text { redacta una } \\
\text { explicación } \\
\text { congruente. }\end{array}$ \\
\hline $\begin{array}{l}\text { Estado del } \\
\text { arte. }\end{array}$ & $\begin{array}{l}\text { El estudiante } \\
\text { no elabora la } \\
\text { revisión del } \\
\text { estado del } \\
\text { arte. }\end{array}$ & $\begin{array}{l}\text { El estudiante } \\
\text { elabora la revisión } \\
\text { del estado del arte } \\
\text { sólo acudiendo a } \\
\text { libros. }\end{array}$ & $\begin{array}{l}\text { El estudiante } \\
\text { elabora la revisión } \\
\text { del estado del arte } \\
\text { sólo acudiendo a } \\
\text { revistas } \\
\text { científicas. }\end{array}$ & $\begin{array}{l}\text { El estudiante } \\
\text { elabora la revisión } \\
\text { del estado del arte } \\
\text { acudiendo a libros y } \\
\text { revistas científicas. }\end{array}$ \\
\hline $\begin{array}{l}\text { Problema de } \\
\text { investigación. }\end{array}$ & $\begin{array}{l}\text { El estudiante } \\
\text { no genera } \\
\text { problema de } \\
\text { investigación. }\end{array}$ & $\begin{array}{l}\text { El estudiante } \\
\text { genera } \\
\text { problema un } \\
\text { investigación pero } \\
\text { no se aprecia } \\
\text { relación con la } \\
\text { línea } \\
\text { investigación de la } \\
\text { universidad. }\end{array}$ & $\begin{array}{l}\text { El estudiante } \\
\text { genera un } \\
\text { problema de } \\
\text { investigación y se } \\
\text { aprecia relación } \\
\text { con la línea de } \\
\text { investigación de la } \\
\text { universidad. }\end{array}$ & $\begin{array}{l}\text { El estudiante genera } \\
\text { un problema de } \\
\text { investigación, } \\
\text { general } \\
\text { específicos, } \\
\text { relacionados con la } \\
\text { línea de } \\
\text { investigación. }\end{array}$ \\
\hline $\begin{array}{l}\text { Objetivos de } \\
\text { investigación. }\end{array}$ & $\begin{array}{l}\text { El estudiante } \\
\text { no identifica } \\
\text { objetivos de } \\
\text { investigación. }\end{array}$ & $\begin{array}{l}\text { El estudiante } \\
\text { identifica } \\
\text { objetivos de } \\
\text { investigación pero } \\
\text { no se aprecia } \\
\text { coherencia con el } \\
\text { problema de } \\
\text { investigación. }\end{array}$ & $\begin{array}{l}\text { El estudiante } \\
\text { identifica } \\
\text { objetivos de } \\
\text { investigación y se } \\
\text { aprecia coherencia } \\
\text { con el problema } \\
\text { de investigación. }\end{array}$ & $\begin{array}{l}\text { El estudiante } \\
\text { identifica objetivos } \\
\text { de investigación, se } \\
\text { aprecia coherencia } \\
\text { con el problema de } \\
\text { investigación, } \\
\text { general } \\
\text { específicos. }\end{array}$ \\
\hline $\begin{array}{l}\text { Hipótesis de } \\
\text { investigación. }\end{array}$ & $\begin{array}{l}\text { El estudiante } \\
\text { no plantea } \\
\text { hipótesis de } \\
\text { investigación. }\end{array}$ & $\begin{array}{l}\text { El estudiante } \\
\text { plantea hipótesis } \\
\text { de investigación } \\
\text { pero no se aprecia } \\
\text { coherencia con el } \\
\text { problema de } \\
\text { investigación. }\end{array}$ & $\begin{array}{l}\text { El estudiante } \\
\text { plantea hipótesis } \\
\text { de investigación y } \\
\text { se aprecia } \\
\text { coherencia con el } \\
\text { problema de } \\
\text { investigación. }\end{array}$ & $\begin{array}{l}\text { El estudiante } \\
\text { plantea hipótesis de } \\
\text { investigación y se } \\
\text { aprecia coherencia } \\
\text { con el problema de } \\
\text { investigación, a } \\
\text { nivel general y } \\
\text { específico. }\end{array}$ \\
\hline
\end{tabular}


REVISTA DE LA UNIVERSIDAD DEL ZULIA. 3a época. Año $11 \mathrm{~N}^{\circ}$ 31, 2020

María del Pilar Quezada-Castro et al /// Desarrollo de competencias investigativas... 421-432

DOI: http://dx.doi.org/10.46925//rdluz.31.26

\begin{tabular}{|c|c|c|c|c|}
\hline $\begin{array}{l}\text { Realidad } \\
\text { problemática. }\end{array}$ & $\begin{array}{l}\text { El estudiante } \\
\text { no redacta la } \\
\text { descripción de } \\
\text { la realidad } \\
\text { problemática. }\end{array}$ & $\begin{array}{l}\text { El estudiante } \\
\text { redacta la realidad } \\
\text { problemática sin } \\
\text { consistencia en los } \\
\text { párrafos. }\end{array}$ & $\begin{array}{l}\text { El estudiante } \\
\text { redacta la realidad } \\
\text { problemática con } \\
\text { consistencia en los } \\
\text { párrafos, citando } \\
\text { datos estadísticos. }\end{array}$ & $\begin{array}{l}\text { El estudiante } \\
\text { redacta la realidad } \\
\text { problemática con } \\
\text { consistencia en los } \\
\text { párrafos, cita datos } \\
\text { estadísticos } \\
\text { consigna la } \\
\text { perspectiva nacional } \\
\text { e internacional }\end{array}$ \\
\hline $\begin{array}{l}\text { Antecedentes } \\
\text { de } \\
\text { investigación. }\end{array}$ & $\begin{array}{l}\text { El estudiante } \\
\text { no redacta } \\
\text { antecedentes } \\
\text { de } \\
\text { investigación. }\end{array}$ & $\begin{array}{lr}\text { El estudiante } \\
\text { redacta } \\
\text { antecedentes } \\
\text { investigación } \\
\text { justificar } \\
\text { incorporación } \\
\text { el pu } \\
\text { en proyecto de } \\
\text { tesis. }\end{array}$ & $\begin{array}{lr}\text { El estudiante } \\
\text { redacta } & \\
\text { antecedentes } & \text { de } \\
\text { investigación } & y \\
\text { justifica } & \text { su } \\
\text { incorporación en } & \text { en } \\
\text { el proyecto } & \text { de } \\
\text { tesis. } & \end{array}$ & $\begin{array}{l}\text { El estudiante } \\
\text { redacta } \\
\text { antecedentes de } \\
\text { investigación, } \\
\text { justifica su } \\
\text { incorporación en el } \\
\text { proyecto de tesis, } \\
\text { ordenándolos } \\
\text { cronológicamente }\end{array}$ \\
\hline $\begin{array}{l}\text { Cumplimiento } \\
\text { de avances } \\
\text { semanales. }\end{array}$ & $\begin{array}{l}\text { El estudiante } \\
\text { no cumple con } \\
\text { los avances } \\
\text { semanales } \\
\text { requeridos por } \\
\text { el docente. }\end{array}$ & $\begin{array}{l}\text { El estudiante } \\
\text { cumple } \\
\text { tardíamente con } \\
\text { los avances } \\
\text { semanales } \\
\text { requeridos por el } \\
\text { docente. }\end{array}$ & $\begin{array}{l}\text { El estudiante } \\
\text { cumple } \\
\text { puntualmente con } \\
\text { los avances } \\
\text { semanales } \\
\text { requeridos por el } \\
\text { docente. }\end{array}$ & $\begin{array}{l}\text { El estudiante } \\
\text { cumple } \\
\text { puntualmente con } \\
\text { los avances } \\
\text { semanales } \\
\text { requeridos por el } \\
\text { docente, } \\
\text { participando de } \\
\text { forma activa ante } \\
\text { cualquier } \\
\text { observación } \\
\text { recomendación. }\end{array}$ \\
\hline
\end{tabular}

\section{Discusión y resultados}

Se identificaron las siguientes competencias:

- Analizar la realidad: Esta competencia le permitirá al estudiante de Derecho observar con sentido crítico las diferentes áreas de desenvolvimiento y desarrollo del ser humano; para lograr ello es factible utilizar como base la jurisprudencia, doctrina, costumbre, legislación o principios generales, fuentes que respaldarán el cuestionamiento ante la deficiencia de la ley. En la rúbrica diseñada, lo descrito se concentra en el criterio de evaluación denominado "ideas de investigación", el mismo que incluye desde la explicación oral congruente hasta la redacción de la misma. Se debe precisar que esta competencia podría ser 
desarrollada desde las diferentes asignaturas que comprenden la carrera profesional en comentario, toda vez que la práctica constante originará el perfeccionamiento y mejora continua que se requiere en la labor como futuro abogado.

- Elaborar un esquema para la búsqueda de información: Esta competencia se caracteriza por la determinación previa de un esquema que le pemitirá al estudiante de Derecho desarrollar un camino por donde transcurrir y no sentirse "a ciegas". Es recomendable que en esta etapa sea acompañado por un tutor o asesor, quien deberá incentivar y motivar la lectura analítica, seleccionando aquellos temas que se vinculan con la idea de investigación. En la rúbrica diseñada, lo descrito se concentra en el criterio de evaluación denominado "estado del arte", el mismo que incluye la revisión de libros y revistas científicas en su primer orden, toda vez que debe contener información relevante que podría ser útil para la continuación del proyecto de investigación.

- Seleccionar los problemas de investigación: Esta competencia se caracteriza porque el estudiante de Derecho deberá priorizar, desde su percepción, cuál es el problema de la realidad pasible de ser solucionado de manera urgente, para ello es importante determinar la fundamentación teórica que respalde su argumento. Aunque no existe un número de problemas de investigación es recomendable segmentar este item en problema general y específicos, los cuales presentarán coherencia y congruencia entre sí. En la rúbrica diseñada, lo descrito se concentra en el criterio de evaluación denominado "problema de investigación", el mismo que incluye la relación entre el problema de investigación seleccionado con una línea de investigación de la universidad, siendo ello un requisito indispensable de todo centro superior de estudios.

- Identificar objetivos de investigación: Esta competencia se caracteriza porque el estudiante de Derecho deberá establecer la congruencia entre entre el objetivo y el problema de investigación seleccionado; es pertinente precisar que los objetivos se individualizan con la presencia de un verbo que marque el sentido de la labor del investigador. En la rúbrica diseñada, lo descrito se concentra en el criterio de evaluación denominado "objetivos de 
investigación", el mismo que incluye la coherencia entre el objetivo y problemas investigación, a nivel general y específico.

- $\quad$ Plantear hipótesis de investigación: Esta competencia se caracteriza porque el estudiante de Derecho deberá, a partir de la revisión del estado del arte, plantear una hipótesis de investigación, la cual tiene por finalidad establecer una relación de coherencia y congruencia con el problema de investigación, ello permitirá corroborar la misma, por ejemplo, en el trabajo de campo. En la rúbrica diseñada, lo descrito se concentra en el criterio de evaluación denominado "hipótesis de investigación", el cual incluye reconocer la coherencia en la redacción final del texto.

- $\quad$ Describir la realidad problemática: Esta competencia se caracteriza porque el estudiante de Derecho deberá redactar de forma clara y sencilla desde la perspectiva general hacia una específica, siendo recomendable consignar las variables de investigación. En la rúbrica diseñada, lo descrito se concentra en el criterio de evaluación denominado "realidad problemática”, el cual incluye la consistencia entre párrafos, la citación de datos de estadísticos y la coyuntura nacional es internacional relacionada con el problema de investigación.

- Redactar los antecedentes de investigación: Esta competencia se caracteriza porque el estudiante de Derecho deberá justificar la presencia de cada antecedente nacional e internacional consignado, no se trata de completar un espacio o requisito del proyecto de investigación, este item representa establecer la necesidad de seleccionar y priorizar los antecedentes atendiendo a un orden cronológico; de lograr ello, otorgará seguridad en la redacción de los antecedentes de investigación. En la rúbrica diseñada, lo descrito se concentra en el criterio de evaluación denominado "antecedentes de investigación".

- Gestionar el tiempo dedicado a la investigación: Esta competencia se caracteriza porque el estudiante de Derecho deberá establecer metas de cumplmiento a corto y mediano plazo, las cuales le permitirán gestionar su tiempo para el desarrollo de los distingos segmentos que comprende el proyecto de investigación. En la rúbrica diseñada, lo descrito se concentra en el criterio de evaluación denominado "cumplimiento de avances semanales". 
REVISTA DE LA UNIVERSIDAD DEL ZULIA. 3a época. Año $11 \mathrm{~N}^{\circ}$ 31, 2020 María del Pilar Quezada-Castro et al /// Desarrollo de competencias investigativas... 421-432

DOI: http://dx.doi.org/10.46925//rdluz.31.26

Asimismo, una vez establecidas las competencias investigativas, corresponde mencionar la proyección de los resultados al aplicar la rúbrica en el estudiante de Derecho:

1. Formar estudiantes con pensamiento crítico del contexto actual.

2. Promover la cultura científica en las facultades de Derecho de las universidades peruanas.

3. Consolidar los semilleros de investigación con tendencia multidisciplinaria.

4. Inteorizar el proceso de formación de investigadores desde las aulas universitarias.

\section{Conclusiones}

La rúbrica sí contribuiría al desarrollo de competencias investigativas del estudiante de derecho, lo cual podría incentivar la producción científica desde las aulas universitarias.

El diseño de la rúbrica holística es ideal para el estudiante de derecho que inicia la asignatura de metodología de la investigación o tesis, lo cual permitirá medir los avances de su proceso de formación, a través de la autoevaluación.

El desarrollo de competencias investigativas del estudiante de Derecho promovería el pensamiento crítico y cultura científica, beneficiándolo en su desenvolvimiento profesional.

Conflictos de intereses

Los autores declaran no tener conflictos de intereses.

\section{Referencias}

Alcón, M. (2016). La rúbrica como instrumento de evaluación en los estudios universitarios. Revista Educar, 10(1), 1-15.

Alcón, M., Menéndez, J. L. y Arbesú, I. (2017). “Closing the Loop”: rúbricas en la evaluación de programas académicos. Revista Observar, 11(2), 115-130.

Alsina, A. \& Rorua, D. (2017). Estableciendo niveles de adquisición de conocimientos matemáticos informales antes de los 3 años: diseño, construcción y validación de una rúbrica. Revista Edma 0-6: Educación Matemática en la Infancia, 6(1), 32-52. 
Araque-Marín, P., Torijano-Gutiérrez, S. A. y Arango-Londoño, N. (2019). Diseño e implementación de rúbricas como instrumento de evaluación del curso de Química General e inorgánica para estudiantes de ingeniería. Revista EIA, 16(31), 131-143.

Blanco, N., Ugarte, Y., Betancourt, Y., Domínguez, I. C. y Bassas, D. (2019). Momentos didácticos para el desarrollo de habilidades investigativas desde la educación en el trabajo. Revista Educación Médica Superior, 33(3), 1-14.

Castañeda, L., Esteve, F. y Adell, J. (2018). ¿Por qué es necesario repensar la competencia docente para el mundo digital? Revista de Educación a Distancia (RED), 18(56), 1-20.

Cruz-Pérez, M. A., Pozo-Vinueza, M. A., Chamorro-Sevilla, H. E. y Urquizo-Buenaño, G. J. (2019). Estrategia didáctica para el desarrollo de habilidades investigativas con el aprovechamento de las TIC. Revista Ciencias Pedagógicas e Innovación, 7(1), 78-85.

Espinoza, E., Rivera, A. R. y Tinoco, N. P. (2016). Formación de competencias investigativas en los estudiantes universitarios. Revista Atenas, 1(33), 1-13.

García-Gutiérrez, Z. y Aznar-Díaz, I. (2019). El desarrollo de competencias investigativas, una alernativa para formar profesionales en pedagogía infantil como personal docente investigador. Revista Electrónica Educare, 23(1), 1-22.

García, N., Paca, N. K., Arista, S. M., Valdez, B. B. y Gómez, I. I. (2018). Investigación formativa en el desarrollo de habilidades comunicativas e investigativas. Revista de Investigaciones Altoandinas, 20(1), 125-136.

Gonzáles, R. y Otero, C. A. (2019). Relación pedagógica tutor-alumno: factor clave para promover las habilidades investigativas. Revista Conrado, 15(70), 48-52.

Lorenzo, Y., Díaz, T. y Gil, A. (2017). El proceso de formación de habilidades investigativolaborales en los estudiantes de la carrera de Contabilidad y Finanzas. Revista Cubana Educación Superior, 2, 157-168.

Martínez, S. F., Medina, F. R. y Salazar, L. A. (2018). Desarrollo de competencias investigativas en los estudiantes. Revista Opuntia brava, 10(1), 336-341.

Masero, I. C., Camacho, M. E. y Vázquez, M. J. (2018). Cómo evaluar conocimientos y competencias en la resolución matemática de problemas en el contexto económico a través de rúbricas. Revista Electrónica Interuniversitaria de Formación del Profesorado, 21(1), 5l-64.

Menéndez, J. L. y Gregori, E. (2016). Las rúbricas en la valoración de las guías docentes. Reflexiones sobre su impacto en la mejora educativa. Revista Observar, 10(1), 84-102. 
Michalón, R. A., Tobar, D. V. y Reinoso, A. P. (2019). Las habilidades investigativas en la carrera de Odontología. Revista Conrado, 15(69), 201-208.

Navarro, M., Navarro, R. E. y García, R. I. (2019). Rúbrica para evaluar ambientes virtuales de aprendizaje. Revista 3C TIC. Cuadernos de Desarrollo Aplicados a las TIC, 7(3), 80-97.

Pacheco, O., Martínez, J. E., López, E. y García, L. F. (2019). Disño y validez de una rúbrica para evaluar las prácticas curriculares en instituciones de educación superior. Revista Espacios, 40(29), 20-27.

Parra, L. S., Marsollier, R. G. y Difabio, H. (2019). Diferencias y relaciones en las habilidades y competencias investigativas de los licenciados en Colombia. Revista de Investigación Educativa, 10(19), 67-74.

Quezada, G. A., Castro, M., Gallo, C. I. y Quezada, M. P. (2020). Deontología como estructura morfológica de la formación en investigación de estudiantes universitarios. Revista Universidad, Ciencia y Tecnología, 24(98), 13-18.

Rodríguez, J. L., Navarrete, Y., Estrada, O. y Vera, L. V. (2019). Valoraciones acerca de la relación entre competencias profesionales y las investigativas. Revista Cubana de Educación Superior, 38(1), 1-11.

Rodríguez-Miñambres, P., Rico, A., López, A. y Ugalde, A. I. (2018). Cómo evaluar una simulación de la metodología por proyectos a través del uso de rúbricas en el Grado de Educación Primaria. Revista Electrónica Interuniversitaria de Formación del Profesorado, 21(2), 43-63.

Segredo, A. M., Reyes, D., Quintana, M. L., Díaz, M., García, I. y Díaz, L. (2017). Desarrollo de habilidades investigativas en el campo de la Salud Pública. Revista Educación Médica Superior, 31(1), 26-47.

Urias, C. M., Rodríguez, C. L. y Zárate, N. E. (2018). Rúbrica para evaluar presentaciones de casos clínicos: instrumento que orienta la calidad en el desempeño. Revista Acta Universitaria, 28(6), 93-100.

Vera-Rivero, D. A., Chirino-Sánchez, L., Blanco, N., Ferrer, L. y Machado, D. L. (2019). Habilidades, motivaciones e interés por la investigación en alumnos ayudantes en Villa Clara. Revista Edumecentro, 11(2), 117-130.

Veytia, M. G., Gómez, J. y Morales, M. B. (2019). Competencias investigativas y mediación tecnológica en doctorandos de Iberoamérica. Revista Internacional de Investigación e Innovación Educativa, 12, 1-19. 